\title{
INCIDENCIA DE LA PROPIEDAD INTELECTUAL EN EL DESARROLLO NACIONAL Y EMPRESARIAL EN EL CONTEXTO DE GLOBLIZACIÓN ACTUAL
}

\author{
STALIN BALLESTEROS GARCÍA* \\ JORGE BULLA DE LA HOZ**
}

La propiedad intelectual, en términos básicos, se refiere a la protección de las creaciones de la mente para obtener de este modo un reconocimiento que bien puede ser económico u honorífico. En el campo de los negocios internacionales anteriormente este concepto no desempeñaba un papel muy importante entre los focos de atención; sin embargo, debido al auge incontenible de la globalización y al tremendo dinamismo empresarial observado actualmente, la propiedad intelectual y sus respectivos estudios obtienen cada vez más relevancia debido a que esta puede ser empleada como una nueva forma de competir y diferenciarse tanto por países como por empresas nacionales y transnacionales.

\section{Historia y EVOLUCiÓN DE LA PROPIEDAD INTELECTUAL}

A lo largo de los años el deseo de crear e innovar ha sido una de las características de la especie humana, características que permitieron la evolución de las sociedades primitivas hasta llegar a las que se observan actualmente. Sin embargo, no fue sino en el siglo XVIII cuando la sociedad internacional moderna se vio en la necesidad de establecer un sistema que protegiera, en términos de propiedad intelectual, al creador y a su invento. Antes de aquella época era difícil que los diferentes actores representativos de la industria de la innovación y la creación confiaran en reuniones internacionales para discutir dicha problemática, tal como lo afirma la OMPI (s.

\footnotetext{
* Profesional en Comercio Internacional de la Universidad Cooperativa de Colombia, magíster en Relaciones Internacionales de la Pontificia Universidad Javeriana y docente de tiempo completo de la Universidad del Magdalena, Santa Marta, Colombia. Contacto: [ballesteros.stalin@gmail.com].

** Estudiante de grado del programa de Negocios Internacionales de la Universidad del Magdalena, Santa Marta, Colombia. Contacto: [jorgeabulla@gmail.com]. Fecha de recepción: 4 de septiembre de 2016. Fecha de aceptación: 30 de noviembre de 2016. Para citar el artículo: Ballesteros García, S. y De la Hoz Bulla, J. Incidencia de la propiedad intelectual en el desarrollo nacional y empresarial en el contexto de globlización actual. Revista La Propiedad Inmaterial n.o 22, Universidad Externado de Colombia, juliodiciembre 2016, pp. 5-18. DOI: http://dx.doi.org/10.18601/16571959.n22.01.
} 
f.): "La necesidad de protección internacional de la propiedad intelectual se hizo patente en 1873, con ocasión de la Exposición Internacional de Invenciones de Viena, a la que se negaron a asistir algunos expositores extranjeros por miedo a que les robaran las ideas para explotarlas comercialmente en otros países".

Contrario a lo que se podría creer, este traspié, en lugar de obstaculizar la creación de un Sistema de Protección de Propiedad Intelectual, catalizó la imperiosa necesidad de establecerlo, y fue así como diez años después, en 1883, dicho sistema empezó a cobrar vida con el Convenio de París para la Protección de la Propiedad Industrial, en el cual quedaron cubiertas las invenciones (patentes) y los dibujos y modelos industriales (ompI, s. f.). Tres años después, en el Convenio de Berna para la Protección de Obras Artísticas y Literarias, se trataron los temas faltantes que dieron lugar al establecimiento de la protección de las mismas con el objetivo de reconocer a los creadores, en el plano internacional, el derecho a controlar el uso de sus obras creativas y a recibir un pago por ese uso (ompI, s. f.). A partir de entonces, y una vez consolidado el sistema internacional, comenzó un gran período de expansión durante el cual se estrechaban las relaciones internacionales entre Estados, haciendo de esta manera que diversas naciones fueran adhiriendo explícita o tácitamente a las pautas internacionales (Roffe, 1987). El creciente número de adhesiones al nuevo sistema concerniente a la propiedad intelectual denota la relevancia del conocimiento y las creaciones de la mente en los diferentes territorios.

Con la llegada del nuevo siglo se observa el periodo comprendido entre las dos guerras mundiales que tiene como desenlace un conflicto entre las dos potencias ganadoras: Estados Unidos y la Unión Soviética. Estos dos países, por varias décadas, intentan someter al otro mediante una guerra armamentista, en un constante vaivén en el cual la capacidad de transformar intangibles (conocimiento, creatividad) en tangibles (nuevas armas, nuevos diseños, nuevas estrategias) dictó el camino y la resolución de dicho conflicto. Es entonces en este momento de la historia cuando la propiedad intelectual y el conocimiento se marcan como una manera importante, valiosa y aun infravalorada de obtener poder.

No obstante dicha importancia, en la práctica, la propiedad intelectual y sus derechos se concentraban en aspectos meramente jurídicos y territoriales, y en términos de comercio exterior se usaban solamente al momento de entablar relaciones bilaterales en las cuales se garantizaba la protección recíproca de los derechos intelectuales, aunque sin alcanzar términos muy profundos (Roffe, 1987). Se puede observar, sin embargo, que últimamente los países han prestado más atención a este tema en orden a obtener mayores ganancias como producto de las ventajas comparativas que ofrece su región y de las competitivas que ofrece su desarrollo, en este caso, el intelectual.

Se ha tratado de una paulatina evolución de la sociedad y de las formas de concebir el comercio internacional que hizo necesario, para el establecimiento y correcta protección de la propiedad intelectual, el ajuste de a las demandas modernas, permitiendo a los Estados y a las empresas obtener los ingresos esperados mediante 
una actualización de estos sistemas de protección. Es así en este contexto que, en 1994, al lado del nacimiento de la OMC, surgió el Acuerdo sobre los Aspectos de los Derechos de Propiedad Intelectual relacionados con el Comercio (ADPIC), en el que primordialmente se buscó " $[r]$ educir las distorsiones del comercio internacional y los obstáculos al mismo, y teniendo en cuenta la necesidad de fomentar una protección eficaz y adecuada de los derechos de propiedad intelectual y de asegurarse de que las medidas y procedimientos destinados a hacer respetar dichos derechos no se conviertan a su vez en obstáculos al comercio legítimo" (omc, 1994, p. 2).

Hasta la fecha el ADPIC es el sistema de normas bajo las cuales se rige la protección de los derechos de propiedad intelectual, siendo administrado por la OMPI.

\section{Propiedad intelectual y globalización}

En el contexto actual, la escena internacional se encuentra configurada bajo relaciones de estrecha interdependencia entre Estados, y entre estos y empresas transnacionales y nacionales; ello como resultado de la profunda globalización que sufrió el planeta desde la caída del Muro de Berlín en 1989.

Desde ese entonces "la sociedad internacional ha sido [...] un objeto en constante evolución" (Barbe, 1995, p. 266). Evolución que dio lugar a fenómenos como un progresivo dinamismo económico en el cual no solamente los Estados fueron los principales actores, sino que el poder privado ostentado por las empresas transnacionales tomó cada vez más relevancia. La emblemática aparición emergente de la inversión privada se vio reflejada en el aumento del volumen y la variedad de transacciones transfronterizas de bienes y servicios, así como en los rendimientos crecientes de los flujos internacionales de capitales y la simultánea difusión acelerada y generalizada de la tecnología (Fondo Monetario Internacional, 1997), lo que condujo a un solo resultado: una ruptura en la forma de entender $\mathrm{y}$ de generar riqueza.

En este orden de ideas se tiene que, en respuesta al crecimiento exponencial de la tecnología y su consiguiente difusión, han aparecido una nueva manera de crear y brindar bienes y servicios y un nuevo modo de interpretar los flujos internacionales de capitales. Esta manera y este modo se originan en un solo concepto: la propiedad intelectual.

La propiedad intelectual, por lo tanto, añade un nuevo segmento al campo de los bienes y servicios, convirtiendo a los activos intangibles en una nueva forma de generar riqueza a nivel nacional y empresarial, a la vez que el capital intelectual se convierte en una manera de generar y, aún más importante, de medir este patrimonio. Por consiguiente, con el fin de obtener dichas riquezas, estos activos se encuentran bajo unos mecanismos de protección. Es así como se ha afirmado que tales "mecanismos de protección de la propiedad intelectual presentan dos aspectos importantes: por una parte, son formas de apropiación de rentas que generan ganancias monopólicas o cuasi monopólicas para sus titulares y, por la 
otra, son incentivos económicos a la investigación en la medida en que retribuyen al innovador por las inversiones realizadas hasta conseguir que sus innovaciones se conviertan en productos de mercado" (Martínez, 2008, p. 60).

Lo anterior conduce a afirmar que el conocimiento se está convirtiendo en todo un mercado en la escena internacional, y al respecto es preciso entender que la actual reestructuración de la geopolítica mundial está incidiendo en diversas doctrinas, una de las cuales es la propiedad intelectual.

Ante esto se hace menester mencionar también que dicho fenómeno, con el del tiempo, ha venido ganando un impulso muy poderoso, tal como lo mencionan Cimoli y Primi (2008): "Año tras año, las oficinas de patentes reciben un número creciente de solicitudes, y otorgan más patentes. Esta dinámica se registra a escala global, y pese a que las economías líderes en capacidades tecnológicas son las que muestran el mayor dinamismo, la actividad se ha intensificado también en las economías emergentes y en los países en desarrollo" (p. 29).

Es decir, la propiedad intelectual ya está siendo explotada por el mundo entero, teniendo así que en términos de comercio internacional existe todo un mercado que gira en torno a ello, razón por la cual el poder económico entre los países se está viendo afectado. Es por eso que en el contexto internacional este tema, en el curso de los años, ha sido motivo de una disputa importante por generar mayor crecimiento económico, originando la creación del ADPIC, aclarando que estos acuerdos han sido logrados en épocas en las que casi inmediatamente después han aparecido fenómenos revolucionarios como internet y la biotecnología. De este modo, esos fenómenos, junto a otros, no han quedado incluidos dentro de los acuerdos, lo que ha imposibilitado una cobertura adecuada y total del ADPIC. Tal como señala Lander (2001), "dados los recientes y acelerados procesos de globalización que se han producido bajo las condiciones de las economías de mercados, estos acuerdos previos ya no son considerados como suficientes por el capital transnacional [...] $[D]$ ichos acuerdos no resultan suficientes por carecer de instrumentos efectivos para garantizar su estricto cumplimiento" (p. 4).

Añadidas a esto todas las regulaciones insertas en el sistema internacional actual, se encuentra también que existen muchas flexibilidades en el campo de la legitimidad y el uso de esta de una forma eficiente en la escena internacional, puesto que las grandes potencias no creen que los estándares establecidos en los ADPIC sean los más pertinentes a la hora de proteger sus patentes. Como lo expresa Moncayo (2008):

Los países en desarrollo imaginaron inicialmente que el ADPIC constituiría un techo de estándares y exigencias de los derechos de propiedad intelectual. La realidad ha superado esta ilusión, pues varios tratados de libre comercio bilaterales y regionales recientes entre países en desarrollo y países desarrollados, principalmente Estados Unidos y la Unión Europea, establecen estándares de protección más elevados y exigentes que los del ADPIC (p. 121). 
Ello pone, sin lugar a dudas, en tela de juicio la efectividad de las normas actuales con las que se pretende regir los millones de patentes que se registran anualmente en el mundo.

Se tiene de esta manera que la confusión que se vive en términos de cumplimiento de las pautas impuestas por el ADPIC es bastante grande, puesto que no es un tema solamente de delimitar, prohibir u otorgar derechos, sino de entender y asumir la complejidad del mundo que se encuentra en una época de cambios constantes, en el que el poder es el bien más preciado y la política global tiende a girar en torno a este, ocasionando que personas o países, según esta filosofía, puedan valerse de cualquier recurso en aras de acrecentar su poder. Dice al respecto Lander (2001):

Mediante nuevas conceptualizaciones y normas internacionales de protección de la propiedad intelectual que se han venido definiendo en los últimos años, se está produciendo un salto cualitativo en la inserción del saber en las relaciones de dominio neocolonial contemporáneas. En este nuevo contexto global, los saberes no sólo están presentes indirectamentecomo dispositivos de legitimación, sino que inciden inmediata y directamente en el establecimiento de nuevas subordinaciones y nuevas relaciones de dominio y explotación (p. 7).

Lo señalado acarrea que ante una falta de igualdad social en términos de propiedad intelectual, los mayores beneficiados son, como siempre ha sido, los entes con un poder coercitivo mayor que los demás; de estos es el poder, son ellos quienes hacen que persistan las desventajas de países que podrían desempeñarse bien en esta área.

De la mano con esto, adentrarse en fenómenos como las integraciones económicas (sean del grado que sean) en todo el mundo y el papel que juegan estos factores en el comercio internacional es también un asunto de gran relevancia para los académicos en términos de comercio exterior. En este orden de ideas se observa que respecto de los tratados de libre comercio se plantean muchos desafíos a la hora de ser firmados, esto debido a los muy altos estándares que poseen algunos países para negociar los términos y el flujo de patentes, y, obviamente, para establecer cuál país se verá más beneficiado; por ejemplo, en el continente americano, los recientes tratados de libre comercio firmados entre países latinoamericanos y Estados Unidos han dado cabida a un proceso de transformación de los regímenes de protección de propiedad intelectual, creando desafíos de política pública que demandan ser abordados en forma sistémica en aras del establecimiento de un nuevo balance entre los incentivos a la innovación y la creación, por una parte, y el interés social de maximizar la difusión del conocimiento y de beneficiar a los consumidores, por otra (Díaz, 2008).

De esta forma queda planteado un debate muy importante a la hora de pensar en involucrar la propiedad intelectual con el comercio internacional, en cuanto a la manera como los países en desarrollo al menos deberían, en los términos 
de negociación de estos tratados de libre comercio, estar en una condición de equidad respecto de los beneficios que obtendrán las grandes potencias con las que se pueda llegar a un pacto de esta índole para así no desmotivar a la industria local.

\title{
3. Propiedad intelectual y su rol en la economia de los estados
}

Uno de los principales focos de atención al momento de estudiar la importancia de la propiedad intelectual en la sociedad internacional es la incidencia que ha tenido esta en términos de crecimiento y desarrollo en las economías de los Estados en los cuales la implementación de procesos y estrategias en pro de obtener el máximo provecho posible es uno de sus principales retos. Según Gould y Gruben (1995), "La política comercial de una nación puede tener implicaciones importantes para establecer una política de propiedad intelectual apropiada. Mientras más abierta sea una economía, mayores serán los beneficios de una protección estricta de los derechos de propiedad intelectual" (p. 267). Lo enunciado hizo pertinente para los Estados, desde épocas en las que la liberalización económica se encontraba en pleno proceso de desarrollo, prestar especial atención a las formas de proteger e implementar sus políticas de propiedad intelectual, esto debido a que "una liberalización del comercio, acompañada de una fuerte protección a la propiedad intelectual, es un importante conductor para el crecimiento económico" (Gould y Gruben, 1995, p. 267).

De aquí se infiere entonces que desde los comienzos del auge del ADPIC en la escena internacional, el impacto resultante de la implementación de políticas de protección de la propiedad intelectual en las economías de los Estados era ya latente en ellos. Casi una década después señala Rozansky (2003):

\begin{abstract}
A nivel de políticas nacionales [...] los cambios para el reconocimiento de los derechos de propiedad intelectual se asocian con mayores accesos al comercio internacional, vía tratados de comercio de carácter multilateral, regional o binacional, y con un incremento en las inversiones extranjeras directas. En cambio, el debilitamiento de la propiedad intelectual se asocia generalmente con políticas de corto alcance (menores costos de la imitación). Las políticas de favorecer el respeto a la propiedad intelectual tienen más relación con el crecimiento en base al estímulo a la innovación, a la incorporación y transferencia de tecnología, y al mejoramiento de la calidad (p. 5).
\end{abstract}

De esta manera, se hace hincapié en la manera como la propiedad intelectual va estrechamente ligada a dos variables: la primera es la participación de los mercados nacionales en el comercio internacional, que afecta el grado de protección que debe poseer un país en aras de fortalecer su economía; y la segunda es la dependencia de esta participación y este desarrollo del establecimiento de estrategias que estimulen la innovación (en todas sus áreas de aplicabilidad). 
En cuanto al grado de afectación, este se debe en gran medida a la nueva competencia en el mercado nacional debido a los evidentes cambios causados por la globalización. Se encuentra entonces que políticas de libre comercio darán lugar a que empresas foráneas entren a nuevos mercados en los que sus métodos tecnológicos de producción serán superiores (Gould y Gruben, 1995). Lo cual deja en evidencia el reto al que se enfrentan las empresas pertenecientes al mercado nacional. Sin embargo, esto, lejos de ser motivo de desánimo para los mercados nacionales, debe ser causal de motivación a cambiar y a mejorar para fijarse en altos estándares de competitividad, y se puede lograr mediante el incentivo de la educación y la innovación en la sociedad en general, debido a que son las personas las que impulsan el desarrollo económico por medio de su ingenio y conocimiento. Y bien, con un mayor crecimiento como resultado de este conocimiento, sin duda habrá una mayor competitividad en distintas ramas de las producciones nacionales, gracias al crecimiento tecnológico que suponen las innovaciones para las economías, siendo esa la premisa clave en cuanto a la relación entre la propiedad intelectual y el desarrollo económico: el auge tecnológico resultante de las innovaciones.

Según Rozanski (2003), "[e]n el Informe Global sobre Competitividad que se prepara para el World Economic Forum de Suiza, ocho son los factores que se toman en cuenta para establecer el índice de competitividad de una economía, uno de ellos es la aplicación de tecnología" (p. 3). Este dinamismo tecnológico, en aras de fortalecer la economía de un país, debe ser sometido a un proceso de protección puesto que "se considera que la verdadera finalidad de la protección del derecho de autor consiste en retribuir el esfuerzo que supone la creación de una obra y la inversión que se hace al producirla y comercializarla" (Silberleib, 2001, p. 3). Esta retribución al esfuerzo es lo que causará el mantenimiento de las innovaciones y, por ende, del crecimiento económico en sí.

Y como segunda parte, dentro de las estrategias que un Estado tome frente a sus posturas de comercio internacional, sin duda estas deben ser cuidadosas puesto que una protección demasiado estricta de los innovadores podría limitar la diseminación de nuevas ideas y, por lo tanto, las oportunidades para el crecimiento económico.

De esta manera se tiene que una excesiva protección sería contraproducente, y sin embargo una posición opuesta también lo sería, debido a que una escasa protección desmotivaría a posibles innovadores puesto que no habría estímulos ya que no se obtendría nada a cambio o fácilmente terceros podrían imitar e incluso robar las ideas ajenas; en efecto, "la fragilidad de la propiedad intelectual [...] favorece actos de piratería" (Pérez, 2007, p. 91). Ello deja claro que los retos en materia de seguridad son grandes y que el problema no es no proteger: la cuestión fundamental es entonces proteger lo suficiente para promover la innovación pero no tanto como para frenarla u obstaculizarla.

1 “Los otros siete son: apertura de la economía, gobierno, finanzas, infraestructura, gerenciamiento, mercado laboral e instituciones” (Rozanski, 2003, p. 3). 
En este orden de ideas no cabe duda de que las políticas de comercio internacional abiertas son beneficiosas para un país en donde una liberalización del comercio acompañada de una adecuada protección a los derechos de propiedad intelectual será un importante conductor en la búsqueda del crecimiento económico.

Cuando un Estado cuente con políticas claras en cuanto a propiedad intelectual será más fácil que el desarrollo económico del país entre en una fase de crecimiento, suponiendo, claro está, que las políticas adoptadas sean correctas. Buitrago (2009) señala al respecto:

Es indudable que la innovación como resultado de los procesos de $I \& D$ es fundamental para el desarrollo de un país y que la mera dependencia industrial, comercial y tecnológica no traerá otra cosa más que dependencia. El desarrollo, por ejemplo, basado exclusivamente en la producción agrí́cola sin un desarrollo industrial y comercio innovativo -no solo tecnológico- de la misma tampoco llevará muy lejos a un país (p. 14).

Y esto es elemental: no basta simplemente con producir o alentar de manera exhaustiva los procesos productivos dentro de un Estado, siendo preciso atender a la manera como se produce, a los métodos y herramientas que se implementan y utilizan para traducir dichos procesos en resultados verdaderamente exitosos. Es en este punto en donde la innovación y la creatividad marcan la ruta del desarrollo tecnológico necesario para ser eficientes en materia de producción y productividad nacional, pero también internacional, porque, como bien señala Mutter (2006), "en este mundo globalizado la interdependencia entre las naciones es cada vez mayor, pero la forma como se plantean las relaciones depende directamente de lo que cada cual tenga para ofrecer" (p. 2). En concordancia con lo citado, la propiedad intelectual afecta desde preceptos internos y privados hasta preceptos globales y abiertos en donde la libre competencia y el mejor uso de la innovación en esta sirven también para impulsar el desarrollo económico. En este orden de ideas es pertinente para cada país tener en cuenta la innovación y la creación como objetos diferenciadores de sus acciones económicas en la escena internacional.

\section{Propiedad intelectual y desarrollo empresarial}

Dentro del sector empresarial hay que tener en cuenta que la propiedad intelectual juega un papel importante para el desarrollo económico y social; proteger el intelecto humano brinda la seguridad necesaria para que las personas continúen creando y así lograr nuevos conceptos e ideas que favorezcan el crecimiento de los países. Es preciso saber que es un hecho cierto que la propiedad intelectual es el camino para el crecimiento de las empresas, haciéndolas mucho más competitivas.

Así entonces, en este sector se tiene los modos de ver el capital que en una sociedad capitalista se ha redirigido hacia la consecución de nuevos espacios no explorados, dentro de los cuales se puedan alcanzar los objetivos trazados mediante 
la utilización y la buena gestión del conocimiento. Respecto de estas proposiciones, Andrade y Martínez (2011) afirman que "[e]l saber y la información como fuerzas de producción, como recursos significativos de una era que se ha dado en llamar post-industrial, están transformando radicalmente los mercados, los servicios, los productos, las economías, la sociedad" (p. 6). De tal posición se deduce que conceptos como el know-how están siendo tenidos muy en cuenta por las empresas al momento de generar productos y servicios eficientes, modernos e innovadores en aras de posicionarlos en mercados tanto internos como externos.

$\mathrm{Y}$ es que la propiedad intelectual va aún más ligada a un concepto que ha venido despertando muchos tipos de intereses en el mundo económico: el capital intelectual. Según esto, el capital intelectual incide de forma determinante en la forma de hacer comercio en este siglo XxI. ¿Por qué? Edvinsson y Malone (1997) explican esta situación comparando a una empresa con un árbol. En este árbol hay una parte visible que son las frutas, y una oculta que son las raíces. Para que dicho árbol pueda seguir dando frutos es necesario que las raíces se encuentren sanas y nutridas. Lo mismo sucede con las empresas: si solo se concentra en los frutos (resultados del tipo que sean) e ignora los valores escondidos, dicha empresa tendrá problemas en el largo plazo.

$\mathrm{Si}$, entonces, el saber y la información están siendo apreciados como fuerzas de producción, el conocimiento está pasando a ser valorado como una nueva forma de capital con la cual Estados y empresas pueden llegar a ser más prósperos y productivos, y la utilización de nuevas herramientas para explotar el conocimiento está permitiendo crear valor dentro y fuera de las organizaciones. Según Simó y Sallan (2008), "el capital intelectual es el conocimiento propiedad de la organización (conocimiento explícito) o de sus miembros (conocimiento tácito) que crea o produce valor presente para la organización” (p. 7). Esto sin duda plantea la importancia del valor del conocimiento y de la adecuada protección de este en el mundo empresarial, ello en orden a lograr ganancias con base en su usufructo.

Se tiene aquí que "[u]na empresa que invierte para desarrollar innovaciones puede rentabilizar esta inversión mediante la comercialización de un producto o proceso novedoso. De este modo, se produce un rendimiento privado de la invención e innovación" (Díaz, 2008, p. 4), e implícitamente esta le aportaría este rendimiento al desarrollo del país en el que se encuentre; es por ello que este debe brindar adecuada protección a tales innovaciones, en aras de evitar hurtos e imitaciones. Tal como lo señala Pérez (2007): "Precisamente la fragilidad de la propiedad intelectual, por su propia naturaleza, favorece actos de piratería" (p. 91). Según este concepto, conduciría a desestimular el crecimiento empresarial, como lo señala Díaz (2008):

Para ninguna empresa privada es incentivo aumentar los conocimientos económicos de la sociedad sin que se produzca un aumento en sus ganancias. Por lo tanto, las empresas, en ausencia de todo sistema de protección de sus inventos (sistema de pa- 
tentes), no esperarían beneficios económicos y no trabajarían para lanzar innovaciones al mercado. Las consecuencias serían muy graves: la sociedad en su conjunto se vería empobrecida y la actividad innovadora de empresas, industrias y otras entidades sería nula o insignificante (p. 4).

Con esto queda más que clara la importancia de fortalecer la propiedad intelectual pero, a su vez, sin otorgar demasiadas licencias, puesto que tal circunstancia podría conducir a la aparición de monopolios. En efecto, " $[s] \mathrm{e}$ ha demostrado que, en ciertas condiciones, un monopolio puede acumular patentes para preservar su poder, permitiendo que éstas 'duerman' a fin de impedir su entrada en una industria” (Gilbert y Newbery, citados por Gould y Gruben, 1995), con lo que se causaría una distorsión en términos de precios o cantidades, como lo señala la OMC (2011): "Hay distorsión del comercio cuando los precios son más elevados o más bajos de lo normal y cuando las cantidades producidas, compradas y vendidas son también superiores o inferiores a lo normal, es decir, a los niveles que existirían normalmente en un mercado competitivo. En los mercados debe ser en un término medio esta protección" (p. 26).

Por lo tanto, resulta menester situar el establecimiento de políticas de protección a la propiedad intelectual en un término medio (sin muchas ni demasiado pocas libertades), orientadas a las necesidades económicas internas de cada país.

\section{Propiedad intelectual y desarrollo en Colombia}

Por último, centraremos nuestra atención en Colombia, país en el cual se observa a simple vista un escenario lleno de retos debido a la escasa existencia de una "conciencia moderna" en la cual se fundamente la búsqueda de crecimiento y desarrollo económico mediante vías nuevas, alternativas y diferentes a las históricamente conocidas, como la del comercio de tangibles y la de la utilización de recursos en aras de buscar el crecimiento mediante preceptos tangibles, dejando a un lado las nuevas maneras no tradicionales de buscar crecimiento mediante los activos intangibles, entre los cuales se encuentra la propiedad intelectual.

Se hace entonces preciso para Colombia (y demás Estados) afrontar un escenario mundial en el que todas las actividades se desenvuelven en un entorno continuamente cambiante y altamente competitivo, en el cual abandonar aquellos conceptos preestablecidos sobre la forma de generar riqueza (sustentados mayormente en preceptos emocionales debido a la resistencia al cambio) y comenzar a dirigir la atención hacia las nuevas corrientes del escenario mundial es la clave para ser competitivos y generar un mayor desarrollo en las distintas esferas de la sociedad (Fujita y Krugman, 2004).

Gómez, Martínez y Arzuaga (2006) señalan acertadamente que "Colombia es un país de contrastes, con abundantes recursos naturales pero con poca capacidad para producir riqueza” (p. 2). Un ejemplo claro que se puede encontrar acerca de 
la riqueza colombiana es su extensa biodiversidad. Pues bien, la riqueza natural se halla es en lo biodiverso, y Colombia se encuentra entre los países más biodiversos del mundo, lo cual le ofrece una ventaja comparativa sobre otros Estados, y sin embargo no es competitivo en este aspecto.

Es precisamente a partir de postulados como este que Colombia necesita diversificar sus manera de producir y de hacer comercio. Al respecto, Mutter (2006) afirma: "Es cierto que en este mundo globalizado la interdependencia entre las naciones es cada vez mayor, pero la forma como se plantean las relaciones depende directamente de lo que cada cual tenga para ofrecer. Esto es evidente en este momento en Colombia. La era en la que una o dos materias primas (exceptuando al petróleo) sostenían un país parece estar llegando a su fin" (p. 86).

Para Colombia es imperativo establecer políticas de nuevas formas de inversión mediante las cuales aspectos como la innovación y la propiedad intelectual le permitan posicionarse competitivamente a nivel local, regional y global. Como bien lo señala De Vivo (2008): "el rezago en que nos encontramos respecto de países como Taiwán y Corea, que hace algo más de 10 años estaban en nuestro mismo grado de desarrollo y que hoy, por cuenta de la innovación y la productividad intelectual, están cruzando la barrera del primer mundo, impone al gobierno una dedicación e interés inminente en este tema (pp. 1-2).

Esto ha venido siendo un jalón de orejas para el gobierno colombiano, el cual regula la protección de la propiedad intelectual mediante la sic, Villamizar (2008) "La Superintendencia de Industria y Comercio (sIC) es la oficina nacional competente en Colombia para el registro y protección de la propiedad industrial" (p. 268). Sin embargo el problema del país radica, más que en la falta de protección, en los obstáculos burocráticos que un innovador encuentra para pasar de la invención al proceso productivo como tal. Esto se observa en las instituciones de educación superior, en donde está, por ejemplo, el caso de Corpoica, que es una entidad que ha obtenido en el sector de biotecnología resultados interesantes en tejidos vegetales, pesticidas y fertilizantes biológicos. Sin embargo, estos resultados se quedan muchas veces como material bibliográfico, en vez ser utilizados para su explotación económica, que sin duda beneficiaría en gran medida al sector agropecuario. Una posible solución a esta problemática sería que Colombia adoptara una ley como la Bayh-Dole de Estados Unidos, que fundamentalmente permite a universidades y centros de investigación explotar comercialmente invenciones obtenidas a partir de financiación estatal, lo que muy probablemente incidiría de manera positiva en los estudiantes y pedagogos que se encuentren adelantando procesos de creación e innovación (Mutter, 2006).

Sin embargo no todo es negativo cuando se observa a un país como Colombia, puesto que una de las características más arraigadas de su población es el deseo de progresar con independencia de las dificultades presentes en el entorno y en el camino hacia el éxito en términos de innovación y propiedad intelectual. Como señala Mutter (2006), "[e]xisten casos de empresas colombianas que han desa- 
rrollado tecnologías propias y han llegado no solamente a distribuir productos protegidos a través de patentes sino que han dado el siguiente paso al licenciar en otros países las tecnologías desarrolladas por ellas (p. 10). De esto se puede inferir que la población colombiana está lista para sumarse a las nuevas formas de ver y producir el capital; falta entonces un mayor compromiso por parte del gobierno nacional para hacerle frente a esta nueva realidad que tiene ante sus ojos.

\section{Conclusión}

La propiedad intelectual y la innovación son dos conceptos y herramientas que permiten a los Estados y a las empresas diferenciarse de sus semejantes, permitiéndoles ser más competitivos debido a que un mayor compromiso en el campo de la innovación permite un mayor auge tecnológico.

En cuanto a los Estados, los mismos deben, como primera medida, establecer políticas comerciales definidas, orientadas a los modelos económicos emergentes, tal como lo es el de los activos, el comercio y la diferenciación mediante intangibles. Sin duda, ello, sumado a una adecuada política de protección de propiedad intelectual, hará a un Estado más competitivo.

En cuanto al sector empresarial, es vital para las empresas mantenerse a la vanguardia del crecimiento intelectual, prestándole especial atención al capital humano de que disponga.

Se puede concluir en cuanto a la propiedad intelectual en Colombia que la noción y el uso de la innovación no son indiferentes para la población, la barrera está entonces en que el gobierno no favorece que personas naturales y jurídicas accedan fácilmente a los sistemas de propiedad intelectual y puedan extraer un provecho económico que sería beneficioso también para el Estado.

Así pues, es de suma importancia tener claro que la propiedad intelectual representa más que un simple requisito; es preciso saber lo que se puede lograr al proteger el conocimiento humano, todo el poder que se puede adquirir al tener una patente y los beneficios que esta trae consigo. Por eso la sociedad moderna debe adaptarse a este proceso de desarrollo que combina lo intelectual con lo social, lo económico, político y cultural, ya que al tratarse de una sociedad que está en crecimiento o que pretende crecer aún más, dicho proceso se hace imperativo en el propósito de alcanzar dichas metas.

\section{REFERENCIAS}

Andrade, R. y Martínez, A. (2013). La creatividad como materia afín a la propiedad intelectual. ¿¿Una tarea pendiente para la ompr? Revista Propiedad Intelectual, 12(16), 168-183. Recuperado de http://www.saber.ula.ve/bitstream/123456789/37610/1/articulo10.pdf

Arzuza, M., Gómez, L. y Martínez, J. (2006). Política pública y creación de em- 
presa en Colombia. Pensamiento \& Gestion, 21, 1-25. Recuperado de http:// rcientificas.uninorte.edu.co/index.php/pensamiento/article/view/3552/2279 BARBE, E. (1995). Relaciones Internacionales. Madrid: Tecnos.

Buitrago, D. (2009). Propiedad intelectual y desarrollo tras el Acuerdo sobre los ADPIC. Revista Propiedad Intelectual, 8(12), 63-90. Recuperado de http://www. redalyc.org/articulo.oa?id=189014944004

Cimoli, M. y Primi, A. (2008). Propiedad intelectual y desarrollo: una interpretación de los (nuevos) mercados del conocimiento. En J. Martínez (ed.), Generación y protección del conocimiento: propiedad intelectual, innovación y desarrollo económico (pp. 29-57). México, D.F.: CEPAL.

De VIVo, S. (2008). Propiedad intelectual y productividad nacional. Revista de Derecho Universidad del Norte, 7(9), 1-3. Recuperado de http://www.scielo. org.co/pdf/dere/n29/n29a01.pdf

DíAz, M. (2008). La propiedad industrial y los sistemas de patentes en el mundo de la información. Acimed, 18(6). Recuperado de http://www.bvs.sld.cu/revistas/ aci/vol18_6_08/aci111208.htm

Edvinsson, L. y Malone, M. S. (1997). El capital intelectual: cómo identificar el valor inexplorado de los recursos intangibles de su empresa. Recuperado de https://books.google.es/books?hl=es\&lr=\&id=lBwT-FT99pAC\&oi=fnd \& $\mathrm{pg}=\mathrm{PR} 11 \& \mathrm{dq}=\mathrm{el}+$ capital + intelectual+edvinsson+malone\&ots=s01ZczeRIn \&sig=3wfkteqcukuoaBqpOf96yc_RIpo\#v=onepage $\& \mathrm{q}=\mathrm{el} \% 20$ capital $\% 20$ intelectual\%20edvinsson $\% 20$ malone $\& \mathrm{f}=$ false

Fondo Monetario Internacional (1997). Informe anual del FMI 1997 - Perspectivas de la economia mundial. Recuperado de https://www.imf.org/external/ pubs/ft/ar/97/s97/pdf/file03.pdf

Gould, D. y Gruben, W. (1995). El papel de los derechos de propiedad intelectual en el crecimiento económico. Economía Mexicana Nueva Epoca 6(2), 265-298. Recuperado de http://www.economiamexicana.cide.edu/num_anteriores/IV2/03_GOULD_265-299.pdf

LANDER, E. (2001). Los derechos de propiedad intelectual en la geopolítica del saber de la sociedad global. Revista del Centro Andino de Estudios Internacionales, 2(2), 1-28. Recuperado de https://www.tni.org/files/Derechos\%20de\%20\%20 propiedad\%20intelectual $\% 20 y \% 20$ geopolítica $\% 20$ del $\% 20$ conocimiento.pdf

Martínez, J. (2008). La protección de los derechos de propiedad intelectual, la innovación y el desarrollo. En J. Martínez (ed.), Generación y protección del conocimiento: propiedad intelectual, innovación y desarrollo económico (pp. 5990). México, D.F.: CEPAL.

MoncaYo, A. (2008). La flexibilidad del ADPIC y su eventual erosión en las negociaciones bilaterales, multilaterales y regionales. En J. Martínez (ed.), Generación y protección del conocimiento: propiedad intelectual, innovación y desarrollo económico (pp. 93-123). México, D.F.: CEPAL.

Mutter, K. (2006). Propiedad Intelectual y desarrollo en Colombia. Revista Es- 
tudios socio-jurídicos, 8(2), 85-101. Recuperado de http://www.scielo.org.co/ pdf/esju/v8n2/v8n2a04.pdf

OMC (1994).ADPIC. Recuperado de https://www.wto.org/spanish/docs_s/legal_s/27trips.pdf

OMPI. Reseña histórica de la OMPI. Recuperado de http://www.wipo.int/about-wipo/ es/history.html

PÉrez, L. (2007). El necesario ajuste entre los derechos de propiedad intelectual y la protección de las medidas tecnológicas. Opinión Jurídica, 6(12), 85-104. Recuperado de http://www.redalyc.org/articulo.oa?id=94501205

Roffe, P. (1987). Evolución e importancia del sistema de la propiedad intelectual. Comercio Exterior, 37(12), 1039-1045. Recuperado de http://revistas. bancomext.gob.mx/rce/magazines/205/5/rce5.pdf

Roffe, P. (2008). Tratados de libre comercio y propiedad intelectual: impactos y desafíos. En J. Martínez (ed.), Generación y protección del conocimiento: propiedad intelectual, innovación y desarrollo económico (pp. 125-158). México, D.F.: CEPAL.

Rozanski, F. (2003). El valor de la propiedad intelectual en los países en desarrollo. Revista INTERCIENCIA, 28(2), 105-110. Recuperado de http://www.interciencia. org/v28_02/rozanski

Silberleib, L. (2001). El derecho la propiedad intelectual y el entorno digital. Información, cultura y sociedad 5, 40-60. Recuperado de http://eprints.rclis. org/16998/

Simó, P. y SAllán, J. (2008). Capital intangible y capital intelectual: revisión, definiciones y líneas de investigación. Estudios de Economia Aplicada, 26(2), 65-78. Recuperado de: http://upcommons.upc.edu/bitstream/ handle/2117/11530/2008-Intangibles-SimoSallan-eea.pdf

Villamizar, S. (2001). Siete principios para un sistema objetivo de Propiedad Intelectual. Revista Estudios socio-jurídicos, 10(1), 265-306. Recuperado de http://www.scielo.org.co/pdf/esju/v10n1/v10n1a9 